\title{
LLL reducing with the most significant bits
}

\author{
Saruchi \\ Indian Institute of Technology New Delhi \\ saruchigoel@gmail.com \\ Damien Stehlé \\ U. Lyon, CNRS, ENS de Lyon, INRIA, UCBL \\ Laboratoire LIP \\ damien.stehle@gmail.com
}

\author{
Ivan Morel \\ U. Lyon, CNRS, ENS de Lyon, INRIA, UCBL \\ U. Sydney, Laboratoire LIP \\ ivan.morel@ens-lyon.org \\ Gilles Villard \\ U. Lyon, CNRS, ENS de Lyon, INRIA, UCBL \\ Laboratoire LIP \\ gilles.villard@ens-lyon.fr
}

\begin{abstract}
Let $B$ be a basis of a Euclidean lattice, and $\widetilde{B}$ an approximation thereof. We give a sufficient condition on the closeness between $\widetilde{B}$ and $B$ so that an LLL-reducing transformation $U$ for $\widetilde{B}$ remains valid for $B$. Further, we analyse an efficient reduction algorithm when $B$ is itself a small deformation of an LLL-reduced basis. Applications include speeding-up reduction by keeping only the most significant bits of $B$, reducing a basis that is only approximately known, and efficiently batching LLL reductions for closely related inputs.
\end{abstract}

\section{Categories and Subject Descriptors}

I.1.2 [Symbolic and Algebraic Manipulation]: Algorithms; F.2.1 [Analysis of Algorithms and Problem Complexity]: Numerical Algorithms and Problems; G.1.3 [Numerical Linear Algebra]: Conditioning

\section{General Terms}

Algorithms

\section{Keywords}

Lattice basis reduction, LLL, floating point arithmetic

\section{INTRODUCTION}

We consider the problem of computing a reducing transformation for a Euclidean lattice basis $B$ using an approximation $\widetilde{B}$ to $B \in \mathbb{R}^{m \times n}$. The necessary background on basis reduction is given in Section 2.2. Our notion of reduction is a variation, robust to perturbations, of the LLL reduction introduced by Lenstra, Lenstra and Lovász in [10]. We assume that the basis vectors are given by the columns of a full column rank matrix $B$. We establish a bound on the closeness between $\widetilde{B}$ and $B$ so that if $U$ is a reducing transformation for $\widetilde{B}$, i.e., the matrix $\widetilde{B} U$ is LLL-reduced, then $B U$ also is reduced.

Permission to make digital or hard copies of all or part of this work for personal or classroom use is granted without fee provided that copies are not made or distributed for profit or commercial advantage and that copies bear this notice and the full citation on the first page. Copyrights for components of this work owned by others than ACM must be honored. Abstracting with credit is permitted. To copy otherwise, or republish, to post on servers or to redistribute to lists, requires prior specific permission and/or a fee. Request permissions from Permissions@acm.org.

ISSAC'14, July 23-25, 2014, Kobe, Japan.

Copyright 2014 ACM 978-1-4503-2501-1/14/07 ...\$15.00.

http://dx.doi.org/10.1145/2608628.2608645.
A main application is to compute a reducing transformation using only a limited number of bits of the input basis, hence possibly at a lower cost than using the entire initial basis. An approach for LLL-reducing a basis $B$ may then be: 1) use the bound and compute an appropriate rounding precision, and deduce an approximation $\widetilde{B}$ sufficiently close to $B ; 2$ ) compute $U$ by reducing $\widetilde{B} ; 3$ ) output $B U$. We follow this general approach for designing Algorithm 1.

We then develop a column scaling strategy for handling cases where the input basis vectors have unbalanced magnitudes. Indeed, in addition to mastering the bit-size of the inputs, dealing with homogeneous magnitudes is often better for lowering the computational cost: as far as we are aware of, no known LLL-reduction algorithm preserves the small bit-size of a "floating-point" basis during the execution, if the magnitudes of the columns differ. A scaling is a pre-processing of the basis $B$ that provides with a "more balanced" matrix $B^{\prime}$ that is also appropriate for computing a reducing transformation. In this purpose we design Algorithm 2 that may be used as Step 2') in the general LLL-reducing scheme above. We note that after both the approximation and the scaling processes, "almost any" LLLreduction algorithm could be used at Step 2) or 2') for computing the reducing transformation.

An important contribution in the study of approximation conditions for preserving reducing transformations has been made by Buchmann in [2]. In the rest of the paper the matrix norm induced by the Euclidean norm is denoted by $\|B\|$. All our vectors will be column vectors, and will be denoted in bold. Buchmann considers an approximation $\widetilde{B}=B+\Delta B$ of absolute precision $p$ to $B$ such that $\|\Delta B\| \leq 2^{-p}$. He provides (see [2, Cor. 4$]$ and its proof) a sufficient condition bound on $p$ to guarantee that if $U$ is such that $(B+\Delta B) U$ is LLL-reduced then $B U$ also has small norm vectors, i.e., within a factor $2^{O(n)}$ of the successive minima of the lattice spanned by $B$ (see Section 2.2 for the definition of the lattice minima). In his analysis, Buchmann relies on the orthogonality defect $\operatorname{od}(B)=\frac{\prod_{i}\left\|\mathbf{b}_{i}\right\|}{\sqrt{\operatorname{det}\left(B^{t} B\right)}}$, and requires a precision $p$ that is logarithmic in the dimension and the orthogonality defect $\operatorname{od}(B)$, and involves the sizes of the successive minima. In terms of relative precision, i.e., for $\Delta B$ such that $\frac{\|\Delta B\|}{\|B\|} \leq 2^{-p}$, the bound is at least logarithmic in the orthogonality defect.

We work with a wider class of approximations: we consider columnwise perturbations of matrices such that $B+$ 
$\Delta B$ satisfies $\max _{i \leq n} \frac{\left\|\Delta \mathbf{b}_{i}\right\|}{\left\|\mathbf{b}_{i}\right\|} \leq \varepsilon$, where $\varepsilon \geq 0$ is small. Hence $B+\Delta B$ is an approximation to $B$ with small columnwise relative precision. In the case of approximate computations, the error bound $\varepsilon$ is of the order of $2^{-p}$, where $p$ is a working precision. Our type of approximations models matrix truncation with small error relatively to each column magnitude. This choice is appropriate for taking into account the backward rounding errors of standard numerical QRfactorization algorithms such as Householder's, that are at the heart of fast reducing algorithms. It also preserves LLLreducedness, as shown in [4]. To introduce our results, we need to define the QR-factorization. Let $B \in \mathbb{R}^{m \times n}$ be full column rank. There exists a unique pair $(Q, R)$ such that

$$
B=Q \cdot R, Q \in \mathbb{R}^{m \times n}, R \in \mathbb{R}^{n \times n},
$$

the columns of $Q$ are orthonormal and the matrix $R$ is uppertriangular with positive diagonal coefficients. The matrices $Q$ and $R$ are respectively called the $Q$-factor and $R$ factor of $B$. For insights into perturbation analysis we may refer the reader to Higham [6], and to [3, 4] in the context of QR-factorization.

Our first result is an improved bound that characterizes which columnwise approximations $B+\Delta B$ to $B$ are allowed such that a reducing transformation $U$ for $B+\Delta B$ remains valid for $B$. Instead of using the defect and the lattice minima, we relate the sufficient precision $1 / \varepsilon$ to the quantity $\operatorname{cond}(R)=\left\||R| \cdot\left|R^{-1}\right|\right\|$. This quantity is defined for any square invertible matrix, and may be viewed as a condition number for the problem of computing $R$. (See, e.g., Zha [19] and Higham [6, Ch. 19].)

In Theorem 1, we show that as soon as

$$
\max _{i \leq n} \frac{\left\|\Delta \mathbf{b}_{i}\right\|}{\left\|\mathbf{b}_{i}\right\|} \leq \frac{1}{c m^{6} \beta^{n} \operatorname{cond}^{2}(R)}
$$

for a constant $c$ that may be made explicit and a constant $\beta$ that can be chosen arbitrarily close to 2 , if $U$ reduces $B+$ $\Delta B$, then $U$ also reduces $B$. Because of the approximation, the matrix $B U$ is LLL-reduced for parameters slightly weaker than those for which $(B+\Delta B) U$ is reduced. However, one may ensure that the parameters are degraded by an arbitrarily small amount (by increasing $c$ ), and the main relevant properties of LLL-reduced bases are preserved, such as those reminded in Lemma 4.

The bound above indicates that taking $p=2 \log \operatorname{cond}(R)+$ $n(1+\epsilon)+O(\log m)$ bits of precision on the input basis $B$ suffices for an arbitrarily small $\epsilon$. We will see, with Lemma 11, that $\operatorname{cond}(R)$ is a more accurate measure than the orthogonality defect $\operatorname{od}(B)$. Indeed, the condition we propose is never more restrictive (up to a $O(\log n)$ additive term) and may be much less so than Buchmann's. In particular, we exhibit a family of bases for which we divide the number of required bits by $\Omega(n)$.

A direct application is an interesting situation considered in [1]. It concerns matrices $B$ whose R-factor satisfies $\left|r_{i j}\right| \leq \eta r_{i i}$ for $\eta \geq 0$ (see Definition 1, with $\theta=0$ ), and for which the ratio $h=\frac{\max r_{i i}}{\min r_{i i}}$ is bounded. In Lemma 12, we show that in this case $\log \operatorname{cond}(B) \lesssim n \log (1+\eta)+\log h$. It follows that $\approx n(1+2 \log (1+\eta))+2 \log h+O(\log m)$ bits of columnwise precision suffice for computing a reducing matrix. The bound is especially interesting when $h \ll \log \|B\|$, such as in [1, Se. 3.2]. The authors of [1] use a constant factor fewer bits than we do (they indeed work with about $\log h$ bits). However, their study is restricted to the first vector of the output basis, which is shown to be no more than a factor $2^{O(n)}$ longer than that of an LLL-reduced basis, compared to the $n$th root of the lattice determinant (see Section 2.2 for the definition of the lattice determinant). This interesting result is an example of transfer between the precision and the quality of the reduction. It also demonstrates the accuracy of our all-purpose bound.

It is essential to note that the ratio $h$ given by the diagonal of $R$ may not be relevant in general for indicating allowed truncations. Indeed, a large $h$ may not imply a large cond $(\cdot)$, as shown by LLL-reduced bases, which can have arbitrarily large ratios $h$ but always satisfy $\operatorname{cond}(R)=2^{O(n)}$ (see Lemma 9).

Our second contribution is an LLL-reduction specifically designed for (floating-point) matrices of the form $B=M_{B}$. $E_{B}$, where $M_{B} \in \mathbb{Z}^{m \times n}$ is full column rank and $E_{B}=$ $\operatorname{diag}_{i}\left(2^{e_{i}}\right)$, with $e_{i} \in \mathbb{Z}$ for all $i$. Such matrices include the ones obtained by columnwise rounding. If the norms of the columns are unbalanced (i.e., the $e_{i}$ 's have different orders of magnitude), the compactness of the representation may be lost when applying an LLL-reduction algorithm to $B$, as the columns get mixed. To circumvent this issue, we propose an algorithm (Algorithm 2) that applies a column scaling $D^{-1}$ to $M_{B} E_{B}$ before calling an LLL-reduction algorithm on $B^{\prime}=M_{B} E_{B} D^{-1}$. The obtained transformation $U$ for $B^{\prime}$ is then mapped to a transformation $D^{-1} U D$ for $B$.

Concerning the correctness of the approach, the main difficulty is to find a scaling $D$ such that $D^{-1} U D$ is unimodular and indeed reduces $B$. This is solved by numerically estimating the diagonal of the R-factor $R$ of $B$ and identifying blocks of consecutive vectors such that $r_{i i} \ll r_{j j}$ if $j$ belongs to a block subsequent to the one of $i$. These blocks are the main source of unbalancedness between the norms of the columns of $B$, and the computed scaling annihilates it. Our algorithm then relies on a "well-behaved" LLL-reduction algorithm that does not destroy the block structure (most known LLL-reduction algorithms are well-behaved, as explained in Section 5.1).

To measure the efficiency of the approach, the relevant quantity is the bit-size of the entries of $B^{\prime}$ once converted to an integer matrix. To estimate it, we view $B=M_{B} E_{B}$ as a distortion $B=\Sigma C$ of an LLL-reduced matrix $C$, where $\Sigma \in$ $\mathbb{R}^{m \times m}$ is non-singular. We then prove, in Theorem 3 , that if $\sigma_{1} \geq \ldots \geq \sigma_{m}$ are the singular values of $\Sigma$, then the bit-size of $B^{\prime}$ is as $O\left(n+\log \left\|M_{B}\right\|+\log \prod_{i \leq\lfloor n / 2\rfloor} \frac{\sigma_{i}}{\sigma_{m-i+1}}\right)$. Several $\Sigma$ 's may exist so that $\Sigma^{-1} B$ is LLL-reduced, and one may optimize the choice of $\Sigma$ to lower $\prod_{i \leq\lfloor n / 2\rfloor} \frac{\sigma_{i}}{\sigma_{m-i+1}}$.

A direct application is to LLL-reduce for strong LLL parameters, a basis that is already reduced for some weak LLL parameters. This is a tempting approach in practice, as LLL-reducing for weak parameters is typically much faster. This strategy is mentioned, e.g., in $[5$, Se. 2.6.1] and $[9$, pp. $70-72] .{ }^{1}$ Here $\Sigma$ is the identity and $\prod_{i \leq\lfloor n / 2\rfloor} \frac{\sigma_{i}}{\sigma_{m-i+1}}=$ 1. Another particular case is $\Sigma=\operatorname{diag}\left(2^{\ell}, 1, \ldots, 1\right)$, for which we have $\prod_{i \leq\lfloor n / 2\rfloor} \frac{\sigma_{i}}{\sigma_{m-i+1}}=2^{\ell}$. This is used in the polynomial factoring algorithm of [7] as well as at the bottom of the recursion in the $\widetilde{\mathrm{L}}^{1}$ algorithm [15].

Finally, our algorithm may be used to batch reductions of

${ }^{1} \mathrm{In}[9]$, the idea is attributed to He, but we could not find the corresponding work. Preliminary results, for strengthening the reducedness, were presented as a poster [11]. 
closely related lattices $\left(L_{k}\right)_{k}$ desribed by bases $\left(B_{k}\right)_{k}$ such that $L_{k+1}=\Sigma_{k} L_{k}$ for all $k$, where the $\Sigma_{k}$ 's have balanced singular values. One may then LLL-reduce $B_{1}$, and, for $k \geq$ 1 , use the transformation matrix $U_{k}$ computed for $B_{k}$, before calling our algorithm on $B_{k+1} U_{k}=\Sigma_{k}\left(B_{k} U_{k}\right)$. This strategy is used, e.g., in communications theory and cryptanalysis applications of LLL $[13,1]$. Our algorithm could prove useful to accelerate and analyse these applications.

Remark. The present work generalizes several results previously investigated for the design of the $\widetilde{\mathrm{L}}^{1}$ algorithm [15]. Perturbation analyses and approximations indeed play a key role since $\widetilde{\mathrm{L}}^{1}$ heavily rely on well-chosen truncations. The results in [15] are essentially focused on LLL-reduced bases, and on specific deformations of such bases. The generalization here is a study with no restrictive assumptions on the initial basis $B$.

Notations. If $B$ is a real-valued matrix, then $|B|$ (resp. $\lfloor B\rfloor)$ is the matrix obtained by replacing each entry of $B$ by its absolute value (resp. the largest integer no greater than it). If $B$ and $B^{\prime}$ are two matrices of identical dimensions, the relation $B \leq B^{\prime}$ must be understood as a componentwise bound. The notations $\|B\|_{F}$ and $\|B\|_{1}$ respectively refer to the Frobenius and induced Manhattan norms of $B$. If $B$ is square and non-singular we define $\kappa(B)=\|B\| \cdot\left\|B^{-1}\right\|$. Clearly, we have $\operatorname{cond}(B) \leq \kappa(B)$. If $\left(x_{i}\right)_{i}$ is a sequence of cardinality $n$, we let $\operatorname{diag}_{i}\left(x_{i}\right)$ denote the $n \times n$ diagonal matrix whose diagonal entries are the $x_{i}$ 's. The computational costs are given in terms of bit operations. We let $\mathcal{M}(t)$ the cost of multiplying two $t$-bit long integers. Finally, all logarithms are in base 2 .

\section{QR AND BASIS REDUCTION}

We extensively rely on roundings and perturbations. We say that $\widetilde{B}$ is an approximation to $B$ of columnwise relative precision $p \geq 0$ if $B-\widetilde{B}=\Delta B$ satisfies $\max _{i} \frac{\left\|\Delta \mathbf{b}_{i}\right\|}{\left\|\mathbf{b}_{i}\right\|} \leq 2^{-p}$.

Approximating real matrices by floating-point ones fits into this context. A precision- $p$ floating-point number is of the shape $m 2^{e}$ with $m$,e integers with $|m| \leq 2^{p}-1$. For any $x \in \mathbb{R}$, there exists $m_{x}, e_{x}$ integers with $\left|m_{x}\right| \leq 2^{p}-1$ such that $\left|m_{x} 2^{e_{x}}-x\right| \leq 2^{-p}|x|$. We call $m_{x} 2^{e_{x}}$ a precision $p$ approximation to $x$. If $x$ is a non-zero integer with known bit-length, such an approximation may be computed in time $O(p+\log (1+\log |x|))$, and $e_{x}$ has bit-length $O(\log \log |x|)$.

An interesting matrix perturbation is columnwise rounding. Let $B=\left(\mathbf{b}_{i}\right)_{i} \in \mathbb{R}^{m \times n}$ be full column rank, and $p$ be a non-negative integer. For each $i \leq n$, let $e_{i}$ be an integer such that $\frac{\left|2^{e_{i}}-\left\|\mathbf{b}_{i}\right\|\right|}{\left\|\mathbf{b}_{i}\right\|}<3 / 4$. Let $E_{B}=2^{-p} \operatorname{diag}_{i}\left(2^{e_{i}}\right)$ and $M_{B}=\left\lfloor B \cdot E_{B}^{-1}\right\rfloor$. Then $\Delta B=B-M_{B} E_{B}$ satisfies $\max _{i \leq n} \frac{\left\|\Delta \mathbf{b}_{i}\right\|}{\left\|\mathbf{b}_{i}\right\|} \leq \frac{\sqrt{m}}{2^{p-2}}$. We may therefore view $M_{B} E_{B}=$ $M_{B} \operatorname{diag}_{i}\left(2^{e_{i}-p}\right)$ as a columnwise floating-point approximation to $B$. Each entry of the mantissa matrix $M_{B}$ is an integer of magnitude smaller than $2^{p+1}$, and the matrix $E_{B}$ that may be represented on $O(n \log \log \|B\|+\log p)$ bits, gives column exponents.

\subsection{Numerical aspects of QR-factorization}

The numerical aspects of QR-factorization have been extensively studied, and we refer the reader to [6, Ch. 19] for a comprehensive entry point to the topic. The following is an explicit variant of classical results.
Lemma 1 ([4, SE. 6]). Let $p \geq 0$ and $B \in \mathbb{R}^{m \times n}$ be non-singular with $R$-factor $R$. Let $\widehat{R}$ be the R-factor computed by Householder's algorithm with floating-point precision $p$. If $80 m n 2^{-p}<1$, then there exists an orthogonal $\widehat{Q}$ such that $\widehat{Q} \widehat{R}=B+\Delta B$ with $\max _{i} \frac{\left\|\Delta \mathbf{b}_{i}\right\|}{\left\|\mathbf{b}_{i}\right\|} \leq 80 m n 2^{-p}$.

Given a matrix $B$, the number of bit operations consumed by Householder's algorithm for computing an approximation to the R-factor of $B$ is $O\left(m n^{2}(\mathcal{M}(p)+\log \log \|B\|)\right)$.

The backward stability lemma above is often combined with a sensitivity result, such as the one below, in order to obtain forward error bounds on the computed quantities.

Lemma 2 (AdAPted From [4, Th. 2.3]). Let B be full column rank in $\mathbb{R}^{m \times n}$, and let $R$ denote its $R$-factor. Let $\Delta B \in \mathbb{R}^{m \times n}$. If $\max _{i \leq n} \frac{\left\|\Delta \mathbf{b}_{i}\right\|}{\left\|\mathbf{b}_{i}\right\|}<1 /(12 m \sqrt{n} \operatorname{cond}(R))$, then $B+\Delta B$ is full column rank and its $R$-factor $R+\Delta R$ satisfies $\left\|\Delta R \cdot R^{-1}\right\|_{F} \leq 6 m \sqrt{n} \operatorname{cond}(R) \max _{i \leq n} \frac{\left\|\Delta \mathbf{b}_{i}\right\|}{\left\|\mathbf{b}_{i}\right\|}$.

Proof. The assertion on the rank follows from [4, Le. 2.2]. From the end of the proof of $[4$, Th. 2.3] with $D=I$, we have $\left\|\Delta R \cdot R^{-1}\right\|_{F} \leq(\sqrt{6}+\sqrt{3}) \sqrt{2} m \sqrt{n} \operatorname{cond}(R) \max _{i} \frac{\left\|\Delta \mathbf{b}_{i}\right\|}{\left\|\mathbf{b}_{i}\right\|}$.

We will also use the following result on the effect on the R-factor of a matrix $B$ of applying a distortion to $B$.

Lemma 3. Let $B \in \mathbb{R}^{m \times n}$ be full column rank and let $R$ denote its $R$-factor. Let $\Sigma \in \mathbb{R}^{m \times m}$ be non-singular, and let $R^{\prime}$ denote the $R$-factor of $\Sigma B$. Then, for all $i$, we have $\left\|\Sigma^{-1}\right\|^{-1} \leq \frac{r_{i i}^{\prime}}{r_{i i}} \leq\|\Sigma\|$.

Proof. The proof is adapted from the proof of [7, Le. 4]. Let $V_{i}(B)=\left\{\mathbf{b}_{i}-\sum_{j<i} y_{j} \mathbf{b}_{j}: y_{1}, \ldots, y_{i-1} \in \mathbb{R}\right\}$. Then $r_{i i}$ is the norm of the shortest vector $\mathbf{b}$ in $V_{i}(B)$. Now, the vector $\Sigma \mathbf{b}$ belongs to $V_{i}(\Sigma B)$. As a result, we have $r_{i i}^{\prime} \leq\|\Sigma \mathbf{b}\| \leq$ $\|\Sigma\| r_{i i}$. The proof that $r_{i i}^{\prime} \geq \frac{r_{i i}}{\left\|\Sigma^{-1}\right\|}$ is analogous.

\subsection{Lattices and LLL basis reduction}

A lattice $L$ is the set of integer combinations of linearly independent vectors in a euclidean space $\mathbb{R}^{n}$ : any lattice may be written as $L=L(B)=B \mathbb{Z}^{n}$, for some full column rank matrix $B \in \mathbb{R}^{m \times n}$. The columns of $B$ are said to form a basis of $L$. If the lattice dimension satisfies $n \geq 2$, the lattice admits infinitely many lattice bases, related by unimodular matrices (i.e., square integer matrices of determinant \pm 1 ): for two full column rank matrices $B, C \in \mathbb{R}^{m \times n}$, we have $B \mathbb{Z}^{n}=C \mathbb{Z}^{n}$ if and only if there exists a unimodular matrix $U$ such that $C=B \cdot U$.

The sparsity of a lattice $L$ may be quantified by its successive minima, defined as $\lambda_{i}(L)=\inf (r: \operatorname{dim}(\operatorname{span} L \cap$ $\mathcal{B}(\mathbf{0}, r)) \geq i)$, for all $i \leq n$. It may also be quantified with the lattice determinant $\operatorname{det} L=\prod_{i} r_{i i}$, where $R$ is the Rfactor of any basis of $L$.

In 1982, Lenstra et al. [10] introduced the notion of LLLreduction of a lattice basis and the LLL-algorithm. If a basis is LLL-reduced, then it is short with respect to the minima of the spanned lattice, and, further, such a basis can be efficiently found using the LLL algorithm. Here we use a variation of LLL-reduction that is more suited to numerical computations.

Definition 1 ([4, Def. 5.3]). Let $\Xi=(\delta, \eta, \theta)$ with $\eta \in$ $(1 / 2,1), \theta \in(0,1]$ and $\delta \in\left(\eta^{2}, 1\right)$. Let $B \in \mathbb{R}^{m \times n}$ be nonsingular with $Q R$ factorization $B=Q \cdot R$. The matrix $B$ is $\Xi$-LLL-reduced if: 
- for all $i<j$, we have $\left|r_{i, j}\right| \leq \eta r_{i, i}+\theta r_{j, j}$ ( $B$ is said size-reduced);

- for all $i$, we have $\delta \cdot r_{i, i}^{2} \leq r_{i, i+1}^{2}+r_{i+1, i+1}^{2}$ ( $B$ is said to satisfy Lovász' conditions).

Let $\Xi=(\delta, \eta, \theta)$ and $\Xi_{\mathrm{w}}=\left(\delta_{\mathrm{w}}, \eta_{\mathrm{w}}, \theta_{\mathrm{w}}\right)$ be valid LLLparameters. We say that $\Xi_{\mathrm{w}}$ is weaker than $\Xi$ and write $\Xi>$ $\Xi_{\mathrm{w}}$ if $\delta>\delta_{\mathrm{w}}, \eta<\eta_{\mathrm{w}}$ and $\theta<\theta_{\mathrm{w}}$. If a basis is $\Xi$-LLL-reduced and if $\Xi>\Xi_{\mathrm{w}}$, then it is also $\Xi_{\mathrm{w}}$-LLL-reduced. This LLLreduction variant is as powerful as the classical definition.

Lemma 4 ([4, TH. 5.4]). Let $B \in \mathbb{R}^{m \times n}$ be $(\delta, \eta, \theta)$ $L L L$-reduced with $R$-factor $R$, for valid parameters $(\delta, \eta, \theta)$. Let $\alpha=\left(\eta \theta+\sqrt{\left(1+\theta^{2}\right) \delta-\eta^{2}}\right) /\left(\delta-\eta^{2}\right)$. Then, for all $i$, $r_{i, i} \leq \alpha \cdot r_{i+1, i+1}$ and $r_{i, i} \leq\left\|\mathbf{b}_{i}\right\| \leq \alpha^{i} \cdot r_{i, i}$. This implies that $\left\|\mathbf{b}_{1}\right\| \leq \alpha^{\frac{n-1}{2}}|\operatorname{det} B|^{1 / n}$ and $\alpha^{i-n} r_{i, i} \leq \lambda_{i}(L(B)) \leq \alpha^{i} r_{i, i}$.

The use of $\Xi$-LLL-reduction rather than the classical definition of LLL-reduction (corresponding to taking $\theta=0$ ) is motivated by the following result. It says that a sufficiently precise approximation to a $\Xi$-LLL-reduced is $\Xi_{\mathrm{w}^{-}}$ LLL-reduced for some $\Xi_{\mathrm{w}}<\Xi$. This result is incorrect if one imposes $\theta_{\mathrm{w}}=0$.

Lemma 5 (Adapted from [4, Cor. 5.7]). For any valid sets of parameters $\Xi=(\delta, \eta, \theta)$ and $\Xi_{\mathrm{w}}=\left(\delta_{\mathrm{w}}, \eta_{\mathrm{w}}, \theta_{\mathrm{w}}\right)$ with $\Xi_{\mathrm{w}}<\Xi$, there exists a constant $c>0$ (that may be made explicit) such that the following holds. For any $\Xi-L L L$ reduced $B \in \mathbb{R}^{m \times n}$ and any $\Delta B$ satisfying $\max _{i} \frac{\left\|\Delta \mathbf{b}_{i}\right\|}{\left\|\mathbf{b}_{i}\right\|} \leq$ $1 /\left(\mathrm{cm}^{2}(1+\eta+\theta)^{n} \alpha^{n}\right)$ where $\alpha$ is as in Lemma 4, the basis $B+\Delta B$ is $\Xi_{\mathrm{w}}-L L L$-reduced.

The $\mathrm{L}^{3}$ algorithm from [10] allows one to compute an LLL-reduced basis of the lattice spanned by a given $B \in$ $\mathbb{Z}^{m \times n}$ in time $O\left(m n^{4} \log ^{2}\|B\| \frac{\mathcal{M}(n+\log \|B\|)}{n+\log \|B\|}\right)$ (see [8]). The $\mathrm{L}^{2}$ and H-LLL algorithms from $[14,12]$ achieve it within $O\left(m \mathcal{M}(n) n^{3}(n+\log \|B\|) \log \|B\|\right)$ bit operations, while the $\widetilde{\mathrm{L}}^{1}$ from [15] runs in time $\widetilde{O}\left(m n^{4}(n+\log \|B\|)\right)$.

Finally, we will use the following generalization of $[15$, Le. 5] to arbitrary bases, which provides a bound on the size of the unimodular matrix between any basis of a lattice and an LLL-reduced basis of the same lattice.

Lemma 6. Let $B \in \mathbb{R}^{m \times n}$ be full column rank. Let $\Xi$ be a valid LLL-parameter, $\alpha$ as in Lemma 4 , and $U$ such that $C=B U$ is $\Xi$-reduced. We have:

$$
\forall i, j:\left|u_{i j}\right| \leq m^{3} \alpha^{n} \operatorname{cond}(R) \cdot \frac{r_{j j}^{\prime}}{r_{i i}}
$$

where $R$ and $R^{\prime}$ respectively denote the $R$-factors of $B$ and $C$.

Proof. Let $B=Q R, C=Q^{\prime} R^{\prime}$ be the QR-factorizations of $B$ and $C$, respectively. Then

$$
U=R^{-1} Q^{t} Q^{\prime} R^{\prime}=\operatorname{diag}_{i}\left(r_{i i}^{-1}\right) \bar{R}^{-1} Q^{t} Q^{\prime} \overline{R^{\prime}} \operatorname{diag}_{i}\left(r_{i i}^{\prime}\right),
$$

with $\bar{R}=R \cdot \operatorname{diag}\left(1 / r_{i i}\right)$ and $\overline{R^{\prime}}=R^{\prime} \cdot \operatorname{diag}\left(1 / r_{i i}^{\prime}\right)$. We have $\left|\bar{R}^{-1}\right| \leq|R|\left|R^{-1}\right| \leq \operatorname{cond}(R) \cdot T$, where $t_{i j}=1$ if $i \leq j$ and $t_{i j}=0$ otherwise. By Lemma 4 , we have $\left|\overline{R^{\prime}}\right| \leq \alpha^{n} T$. We also have $|Q|,\left|Q^{\prime}\right| \leq M$, where $m_{i j}=1$ for all $i, j$. Using the triangular inequality, we obtain:

$$
\begin{aligned}
|U| & \leq \operatorname{cond}(R) \alpha^{n} \cdot \operatorname{diag}\left(r_{i i}^{-1}\right) T M^{t} M T \operatorname{diag}\left(r_{i i}^{\prime}\right) \\
& \leq m n^{2} \alpha^{n} \operatorname{cond}(R) \cdot \operatorname{diag}\left(r_{i i}^{-1}\right) N \operatorname{diag}\left(r_{i i}^{\prime}\right),
\end{aligned}
$$

where $N$ is the all-1 matrix with appropriate dimensions.

\section{WELL-CONDITIONED MATRICES}

As we have seen, the quantity $\operatorname{cond}(\cdot)$ plays a role both for the sensitivity of the R-factor under columnwise perturbations (Lemma 2) and for the size of the unimodular transformation between a lattice basis and an LLL-reduced basis of the same lattice (Lemma 6). It is therefore interesting to investigate sufficient conditions to ensure a small value of $\operatorname{cond}(\cdot)$.

Lemma 7. Let $B \in \mathbb{R}^{m \times n}$ full column rank and $\Sigma \in$ $\mathbb{R}^{m \times m}$ non-singular. Let $R$ and $R^{\prime}$ respectively denote the $R$-factors of $B$ and $\Sigma B$. Then $\operatorname{cond}\left(R^{\prime}\right) \leq m n \kappa(\Sigma) \operatorname{cond}(R)$. Further, if $B$ and $B^{\prime}$ are square and upper-triangular, then $\operatorname{cond}\left(R^{\prime}\right) \leq \kappa(\Sigma) \operatorname{cond}(R)$.

Proof. Write $B=Q R$ (resp. $\Sigma B=Q^{\prime} R^{\prime}$ ), where the columns of $Q$ (resp. $Q^{\prime}$ ) are orthogonal. The first result follows from the equation $\left|R^{\prime}\right|\left|\left(R^{\prime}\right)^{-1}\right|=\left|\left(\left(Q^{\prime}\right)^{T} \Sigma Q\right) R\right|$. $\left|R^{-1}\left(\left(Q^{\prime}\right)^{T} \Sigma Q\right)^{-1}\right|$ and the triangular inequality. The proof simplifies when $Q$ and $Q^{\prime}$ are square, as in that case we have $\left\|\left(Q^{\prime}\right)^{T} \Sigma Q\right\|=\|\Sigma\|$ and $\left\|\left(\left(Q^{\prime}\right)^{T} \Sigma Q\right)^{-1}\right\|=\left\|\Sigma^{-1}\right\|$.

As a corollary, we obtain the fact that a small columnwise perturbation cannot increase cond $(\cdot)$ by much.

Lemma 8. Let $B \in \mathbb{R}^{m \times n}$ be full column rank with $R$ factor $R$, and $\Delta B \in \mathbb{R}^{m \times n}$. If we have $\max _{i} \frac{\left\|\Delta \mathbf{b}_{i}\right\|}{\left\|\mathbf{b}_{i}\right\|}<$ $1 /(12 m \sqrt{n} \operatorname{cond}(R))$, then $B+\Delta B$ is full column rank and its $R$-factor $R+\Delta R$ satisfies $\operatorname{cond}(R+\Delta R) \leq 4 \operatorname{cond}(R)$.

Proof. We write $R+\Delta R=\Sigma R$, with $\Sigma=I+\Delta R$. $R^{-1}$. By Lemma 2 , we know that $\left\|\Delta R \cdot R^{-1}\right\| \leq \| \Delta R$. $R^{-1}\left\|_{F} \leq 6 m \sqrt{n} \operatorname{cond}(R) \max _{i}\right\| \Delta \mathbf{b}_{i}\|/\| \mathbf{b}_{i} \|$. Thanks to the assumption on $\max _{i}\left\|\Delta \mathbf{b}_{i}\right\| /\left\|\mathbf{b}_{i}\right\|$, we obtain that $\Sigma$ is nonsingular. Further $\|\Sigma\| \leq 2$ and $\left\|\Sigma^{-1}\right\| \leq \| I+\sum_{k \geq 1}(\Delta R$. $\left.R^{-1}\right)^{k} \| \leq 2$. As a result, we obtain $\kappa(\Sigma) \leq 4$. Lemma 7 provides the result.

The following result shows that any LLL-reduced basis has a small cond $(\cdot)$.

Lemma 9 ([4, LE. 5.5]). Let $\Xi=(\delta, \eta, \theta)$ be any valid set of LLL-parameters. If $B \in \mathbb{R}^{m \times n}$ is $\Xi$-LLL-reduced and $R$ is its $R$-factor, then $\operatorname{cond}(R) \leq \frac{|1-\eta-\theta| \alpha+1}{(1+\eta+\theta) \alpha-1}((1+\eta+$ $\theta) \alpha)^{n}$, with $\alpha$ as in Lemma 4 .

In fact, LLL-reducedness is a much stronger assumption than needed, for $\operatorname{cond}(R)$ to be bounded as $2^{O(n)}$. A weaker assumption is used in the following result. Note that the assumption is satisfied for LLL-reduced bases, by Lemma 4 applied to square diagonal sub-blocks or the R-factor.

Lemma 10. Let $B \in \mathbb{R}^{m \times n}$ be full column rank with $R$ factor $R$. Assume that there exists $\alpha>1$ such that for all $i \leq j$, we have $\left|r_{i j}\right| \leq \alpha^{j-i+1} r_{j j}$. Then $\operatorname{cond}(R) \leq$ $\frac{\alpha^{2}}{\left(\alpha^{2}-1\right) \sqrt{4 \alpha^{4}-1}}\left(2 \alpha^{3}\right)^{n}$.

Proof. Let $\bar{R}=R \cdot \operatorname{diag}\left(r_{i i}^{-1}\right)$. We have $\operatorname{cond}(R)=$ $\operatorname{cond}(\bar{R}) \leq \kappa(\bar{R})$. A direct computation shows that $\|\bar{R}\| \leq$ $\left(\sum_{i=1}^{n} \sum_{j=1}^{i} \alpha^{2 j}\right)^{1 / 2} \leq\left(\sum_{i=1}^{n} \alpha^{2 i} \cdot \frac{\alpha^{2}}{\alpha^{2}-1}\right)^{1 / 2} \leq \frac{\alpha^{n+2}}{\alpha^{2}-1}$. It now suffices to bound $\left\|\bar{R}^{-1}\right\|$ from above.

Write $\bar{R}=I+M$, where $M$ is the matrix having same elements as $\bar{R}$ but with zeroed diagonal coefficients. We have 
$\bar{R}^{-1}=(I+M)^{-1}=\sum_{0<k<n}(-M)^{k}$. Using the triangle inequality, we obtain that $\left|\bar{R}^{-1}\right| \leq \sum_{0 \leq k<n}|M|^{k}$. Let $J$ denote the $n \times n$ matrix such that $J_{i j} \stackrel{\leq=1}{=}$ if $i-j=1$, and $J_{i j}=0$ otherwise. By assumption, we have $|M| \leq$ $\alpha \sum_{1 \leq k<n}(\alpha J)^{k}=\alpha^{2} J(I-\alpha J)^{-1}$. As a consequence:

$$
\begin{aligned}
\left|\bar{R}^{-1}\right| & \leq \sum_{0 \leq k<n}\left(\alpha^{2} J(I-\alpha J)^{-1}\right)^{k} \\
& =\left(I-\alpha^{2} J(I-\alpha J)^{-1}\right)^{-1} \\
& =(I-\alpha J)\left(I-\left(\alpha+\alpha^{2}\right) J\right)^{-1} \\
& \leq \sum_{0 \leq k<n}\left(\alpha+\alpha^{2}\right)^{k} J^{k} \leq \sum_{0 \leq k<n}\left(2 \alpha^{2}\right)^{k} J^{k} .
\end{aligned}
$$

We derive that $\left\|\bar{R}^{-1}\right\| \leq\left(\sum_{k=0}^{n-1}\left(2 \alpha^{2}\right)^{2 k}\right)^{1 / 2} \leq \frac{\left(2 \alpha^{2}\right)^{n}}{\sqrt{4 \alpha^{4}-1}}$, which leads to the result.

As discussed in the introduction, Buchmann provides in [2] a sufficient bound on the input precision to guarantee the correctness of the algorithm of the next section. The bound is at least logarithmic in the orthogonality defect $\operatorname{od}(B)=$ $\prod_{i} \frac{\left\|\mathbf{b}_{i}\right\|}{r_{i i}}$. of the full column rank matrix $B \in \mathbb{R}^{m \times n}$ with $\mathrm{R}$-factor $R$. Our sufficient condition involves a precision logarithmic in $\operatorname{cond}(B)$. The following lemma reveals the relationship between $\operatorname{cond}(B)$ and $\operatorname{od}(B)$.

Lemma 11. Let $B \in \mathbb{R}^{m \times n}$ be full column rank and $R$ be its $R$-factor. Then for all $i$, we have $\left\|\mathbf{b}_{i}\right\| \leq r_{i i} \operatorname{cond}(R)$, implying that $\operatorname{od}(B) \leq \operatorname{cond}(R)^{n}$. Oppositely, we have cond $(R)$. $n^{-3 / 2} \leq \operatorname{od}(B)$. Finally there exists a sequence of full column rank matrices $B \in \mathbb{R}^{n \times n}$ of growing dimension $n$ such that $\operatorname{od}(B)=\operatorname{cond}(R)^{\Theta(n)}$.

Proof. For each $j \geq i$, the coefficient $(j, i)$ of $|R| \cdot\left|R^{-1}\right|$ is bounded from below by $\left|r_{j i}\right| / r_{i i}$ (when doing the inner product of the $j$ th row of $|R|$ with the $i$ th column of $\left|R^{-1}\right|$, the coefficient $\left|r_{j i}\right|$ is multiplied with $\left.1 / r_{i i}\right)$. This implies that $\frac{\left\|\mathbf{r}_{i}\right\|}{r_{i i}} \leq \operatorname{cond}(R)$ holds for all $i$. Multiplying over varying $i$ gives the first statement.

We now prove the second statement. The coefficient $(i, j)$ of $\left|R^{-1}\right|$ is bounded from above by $\frac{1}{r_{i i}} \cdot \prod_{i<k \leq j} \frac{\left\|\mathbf{b}_{k}\right\|}{r_{k k}}$ (this can be obtained by using the cofactors of $R$ to compute $\left|R^{-1}\right|$ and then applying Hadamard's bound). As a result we obtain that the coefficient $(i, j)$ of $|R| \cdot\left|R^{-1}\right|$ is bounded from above by $\sum_{i \leq k \leq j} \prod_{k \leq \ell \leq j} \frac{\left\|\mathbf{b}_{\ell}\right\|}{r_{\ell \ell}}$, which is itself $\leq n \cdot \operatorname{od}(B)$.

Finally, consider the $n$-dimensional upper triangular matrix $R$ defined by $r_{i j}=\alpha^{j-i+1}$, for $\alpha>1$ arbitrary. By considering only the first row of $R$, we obtain that $\operatorname{od}(R) \geq$ $\alpha^{n(n-1) / 2}$. Lemma 10 allows us to complete the proof.

Another class of bases with relatively small cond(.) is given by upper triangular matrices $B$ whose diagonal entries have balanced magnitudes, and which are size-reduced with $\theta=0$ in Definition 1 . If the largest ratio $h$ between two diagonal entries is small, then as shown by next lemma, the quantity $\operatorname{cond}(B) \leq h 2^{O(n)}$ may be thought as small. (A geometric interpretation is given in [4, Se.3.3].)

Lemma 12. Let $B \in \mathbb{R}^{n \times n}$ be an upper triangular, invertible matrix with the property that for all $i<j$ we have, $\left|b_{i j}\right| \leq \eta\left|b_{i i}\right|$, for some $\eta \geq 0$. Then we have $\operatorname{cond}(B) \leq$ $2 n(1+\eta)^{n-1} \max _{i, j} \frac{\left|b_{i i}\right|}{\left|b_{j j}\right|}$.
Proof. Let $\bar{B}=\operatorname{diag}\left(b_{i i}^{-1}\right) \cdot B$. For all $i<j$ we have $\left|\bar{b}_{i j}\right| \leq \eta \bar{b}_{i i}=\eta$. Therefore, we have $\left|\bar{B}^{-1}\right| \leq T^{-1}$, where $T \in$ $\mathbb{R}^{n \times n}$ is upper triangular with $t_{i i}=1$ and $\bar{t}_{i j}=-\eta$ for $i<j$ (see, e.g., [6, Th. 8.12]). Since $S=T^{-1}$ satisfies $s_{i i}=1$ and $s_{i j}=\eta(1+\eta)^{j-i-1}$ for $i<j$ (see, e.g., [6, Eq. (8.4)]), we obtain $v_{i j}=2 \eta(1+\eta)^{j-i-1}$, for $i<j$, where $V=|T| T^{-1}$. It follows that $|V| \leq 2(1+\eta)^{n-1} M$ where $m_{i j}=1$ for all $i \leq j$, and $m_{i j}=0$ otherwise. Since $|\bar{B}|\left|\bar{B}^{-1}\right| \leq V$, we may now write $\operatorname{cond}(B)=\left\|\operatorname{diag}\left(b_{i i}\right) \cdot V \cdot \operatorname{diag}\left(b_{j j}^{-1}\right)\right\| \leq$ $2(1+\eta)^{n-1}\left\|\left(\frac{b_{i i}}{b_{j j}}\right)_{1 \leq i, j \leq n}\right\|$, which shows the assertion.

Given an invertible $B \in \mathbb{R}^{n \times n}$ with R-factor $R$, one may estimate $\operatorname{cond}(R)$ in the following way. By [17], we have:

$$
\begin{aligned}
\operatorname{cond}(R) & \leq n \operatorname{cond}(B) \leq n^{3 / 2}\left\||B| \cdot\left|B^{-1}\right|\right\|_{1} \\
& \leq n^{3 / 2}\left\|B D^{-1}\right\|_{1}\left\|D B^{-1}\right\|_{1} \\
& \leq n^{5 / 2}\left\|B D^{-1}\right\|\left\|D B^{-1}\right\|,
\end{aligned}
$$

where $D=\operatorname{diag}_{i}\left(\left\|\mathbf{b}_{i}\right\|_{1}\right)$. Therefore, it suffices to find estimates of $\left\|B D^{-1}\right\|$ and $\left\|D B^{-1}\right\|$. We refer the reader to $[6$, Ch. 15] for a presentation of classical approaches for estimating a matrix norm $\|A\|$, such as through a random sampling of vectors $\mathbf{x}_{i}$ for measuring $\max _{i} \frac{\left\|A \mathbf{x}_{i}\right\|}{\left\|\mathbf{x}_{i}\right\|}$. If $B$ is an integer matrix, this results in an algorithm of bit-complexity $\widetilde{O}\left(n^{3} \log \|B\|\right)$ using [18].

\section{REDUCING BY ROUNDING}

Our first main result is Theorem 1 . We analyse the effect of applying to a lattice basis $B$ a transformation matrix $U$ reducing a perturbation $B+\Delta B$ of $B$. We rely on Lemma 13, which shows that a reducing transformation $U$ for a given basis $B$ remains a reducing transformation for any basis sufficiently close to $B$. This result, with a backward stability flavor, is then applied to $B+\Delta B$ for establishing the reducedness of $B$.

LEMma 13. For any valid sets of LLL-parameters $\Xi=$ $(\delta, \eta, \theta)$ and $\Xi_{\mathrm{w}}=\left(\delta_{\mathrm{w}}, \eta_{\mathrm{w}}, \theta_{\mathrm{w}}\right)$ with $\Xi_{\mathrm{w}}<\Xi$, there exists a constant $c>0$ (that may be made explicit) such that the following holds. Let $B \in \mathbb{R}^{m \times n}$ full column rank, $R$ its $R$ factor, and $U$ such that $B U$ is $\Xi-L L L$-reduced. Assume that $\Delta B \in \mathbb{R}^{m \times n}$ satisfies $\max _{i} \frac{\left\|\Delta \mathbf{b}_{i}\right\|}{\left\|\mathbf{b}_{i}\right\|} \leq 1 /\left(\mathrm{cm}^{6} \beta^{n} \operatorname{cond}^{2}(R)\right)$ with $\beta=(1+\eta+\theta) \alpha^{2}$ and $\alpha$ as in Lemma 4. Then $(B+$ $\Delta B) U$ is $\Xi_{\mathrm{w}}$-reduced.

Proof. By Lemma 6, we have $\left|u_{j i}\right| \leq m^{3} \alpha^{n} \operatorname{cond}(R) \cdot \frac{r_{i i}^{\prime}}{r_{j j}}$ for all $i, j$, where $R$ (resp. $R^{\prime}$ ) is the R-factor of $B$ (resp. $C=B U)$. Let $C+\Delta C=(B+\Delta B) U$. We obtain that $\Delta \mathbf{c}_{i}=$ $\sum_{j} u_{j i} \Delta \mathbf{b}_{j}$ satisfies (for all $i$ ):

$$
\left\|\Delta \mathbf{c}_{i}\right\| \leq\left(m^{3} \alpha^{n} \operatorname{cond}(R) \max _{j} \frac{\left\|\Delta \mathbf{b}_{j}\right\|}{\left\|\mathbf{b}_{j}\right\|}\right) \cdot \sum_{j} \frac{r_{i i}^{\prime}}{r_{j j}}\left\|\mathbf{b}_{j}\right\| .
$$

Now, by Lemma 11, we have that $\left\|\mathbf{b}_{j}\right\| / r_{j j} \leq \operatorname{cond}(R)$ holds for all $j$. By using the fact that $r_{i i}^{\prime} \leq\left\|\mathbf{c}_{i}\right\|$, we derive that:

$$
\max _{i} \frac{\left\|\Delta \mathbf{c}_{i}\right\|}{\left\|\mathbf{c}_{i}\right\|} \leq m^{4} \alpha^{n} \operatorname{cond}^{2}(R) \max _{i} \frac{\left\|\Delta \mathbf{b}_{i}\right\|}{\left\|\mathbf{b}_{i}\right\|} .
$$

Applying Lemma 5 to $C$ and $C+\Delta C$ provides the result.

The following result extends [15, Le 7], to any full column rank matrix $B$. 
THEOREM 1. For any valid sets of parameters $\Xi_{\mathrm{w}}<\Xi$, there exists $c>0$ (that may be made explicit) such that the following holds. Let $B \in \mathbb{R}^{m \times n}$ full column rank, $R$ its $R$ factor, and $\Delta B$ satisfying $\max _{i} \frac{\left\|\Delta \mathbf{b}_{i}\right\|}{\left\|\mathbf{b}_{i}\right\|} \leq 1 /\left(\mathrm{cm}^{6} \beta^{n} \operatorname{cond}^{2}(R)\right)$ with $\beta$ as in Lemma 13. Then if $U$ is such that $(B+\Delta B) U$ is $\Xi$-LLL-reduced, then $B U$ is $\Xi_{\mathrm{w}}-L L L$-reduced.

Proof. By Lemma 8 , we have $\operatorname{cond}(R+\Delta R) \leq 4 \operatorname{cond}(R)$, where $R+\Delta R$ is the R-factor of $B+\Delta B$. We conclude by using Lemma 13 on $B+\Delta B$ with perturbation $-\Delta B$, to establish the reducedness of $B=(B+\Delta B)-\Delta B$.

As a corollary of the theorem just above, Algorithm 1 is correct. Note that an upper bound of $\operatorname{cond}(R)$ is required as part of the input, where $R$ denotes the R-factor of $B$. Such a bound may be derived from a priori information on $B$ (e.g., using Lemmata 7, 8 and 9), or may be estimated, as explained at the end of Section 3. At Step 7, any LLLreducing algorithm may be used. In the next section, we describe and analyze an LLL-reducing algorithm specifically designed for floating-point lattice bases $M_{B} E_{B}$, when they are themselves small distortions of LLL-reduced bases.

Input: $B \in \mathbb{R}^{m \times n}$ full column rank; valid LLL-parameters $\Xi_{\mathrm{w}}$; $\chi \geq \operatorname{cond}(R)$, where $R$ is the R-factor of $B$.

Output: $\mathrm{A} \Xi_{\mathrm{w}}$-reduced basis of the lattice spanned by $B$.

1 Choose valid LLL-parameters $\Xi>\Xi_{\mathrm{w}}$.

2 Compute the constants $c$ and $\beta$ of Theorem 1 .

3 Set $p:=\left\lceil\log \left(4 \mathrm{~cm}^{7} \beta^{n} \chi^{2}\right)\right\rceil$.

4 For each $i \leq n$, find $e_{i} \in \mathbb{Z}$ such that $\frac{\left|2^{e_{i}}-\left\|\mathbf{b}_{i}\right\|\right|}{\left\|\mathbf{b}_{i}\right\|} \leq 3 / 4$.

5 Set $E_{B}:=2^{-p} \operatorname{diag}_{i}\left(2^{e_{i}}\right)$.

6 Set $M_{B}:=\left\lfloor B \cdot E_{B}^{-1}\right\rfloor$.

7 Compute $U$ such that $\left(M_{B} E_{B}\right) \cdot U$ is $\Xi$-LLL-reduced. 8 Return $B \cdot U$

Algorithm 1: LLL-reduction of $B$ using a columwise floating-point approximation of $B$

\section{REDUCING BY SCALING}

We now describe and analyze an algorithm for efficiently LLL-reducing floating-point lattice bases $M_{B} E_{B}$, such as the one involved at Step 7 of Algorithm 1. To LLL-reduce the floating-point matrix $M_{B} E_{B}$, we may interpret it as an integer matrix, and LLL-reduce that integer matrix. However, if the exponents are very unbalanced, the bit-size of $M_{B} E_{B}$ as an integer matrix (and hence the cost of the LLL-reduction) may be much higher than the bit-size of $M_{B} E_{B}$ as a floatingpoint matrix. Our algorithm scales the columns of $M_{B} E_{B}$, to obtain a matrix $M_{B} E_{B} D^{-1}$, so that the conversion to an integer matrix essentially preserves the small bit-size of the floating-point representation. The main difficulty to establish the correctness of the algorithm is to ensure that the transformation matrix $U$ when LLL-reducing $M_{B} E_{B} D^{-1}$ is relevant for LLL-reducing $M_{B} E_{B}$ (note that the spanned lattices are different).
Input: $M_{B} \in \mathbb{Z}^{m \times n}$ full column rank;

$E_{B}=2^{-p} \operatorname{diag}_{i}\left(e_{i}\right)$ with $e_{i} \in \mathbb{Z}$ for all $i$; valid LLL-parameters $\Xi=(\delta, \eta, \theta)$;

$\chi \geq \operatorname{cond}(R)$, where $R$ is the R-factor of $M_{B} E_{B}$.

Output: A matrix pair $(U, D)$ such that $D^{-1} U D$ is unimodular, $\left(M_{B} E_{B}\right)\left(D^{-1} U D\right)$ is $\Xi$-LLL-reduced and $D=\operatorname{diag}\left(2^{d_{i}}\right)$ with $d_{i} \in \mathbb{Z}$ for all $i$.

1 Set $p:=10+\left\lceil\log \left(m^{3.5} \chi\right)\right\rceil$.

2 Call Householder's algorithm on $M_{B} E_{B}$ with precision $p$; let $\hat{R}$ be its output.

3 Set $i_{0}:=1$ and $k:=1$.

4 For $i \leq n$, do: If $\left(\min _{j \geq i} \hat{r}_{j j}>(8 / \theta) \cdot \max _{j<i} \hat{r}_{j j}\right)$, then increment $k$ and set $i_{k}:=i$.

5 For all $1<\ell<k$, set $g_{\ell}:=\left(\min _{i_{\ell} \leq i<i_{\ell+1}} \hat{r}_{i i}\right) /\left(\max _{i_{\ell-1} \leq i<i_{\ell}} \hat{r}_{i i}\right)$.

6 For all $1 \leq \ell<k$ and all $i_{\ell} \leq i<i_{\ell+1}$, set $d_{i}:=e_{1}+\sum_{\ell^{\prime}<\ell}\left\lfloor\log \left(g_{\ell^{\prime}} / 4\right)\right\rfloor$.

7 Set $D:=\operatorname{diag}\left(2^{d_{i}}\right) . \quad \quad / *$ Column scaling $* /$

8 Set $\Xi^{\prime}=(\delta, \eta, \theta / 2)$.

9 Compute $U$ s.t. $\left(M_{B} E_{B} D^{-1}\right) \cdot U$ is $\Xi^{\prime}$-LLL-reduced.

10 Return $(U, D)$.

Algorithm 2: LLL-reduction of a floating-point matrix $M_{B} E_{B}$ using column scaling.

Algorithm 2 can be divided into four main parts:

- Finding approximations of the diagonal coefficients of the R-factor of the input basis $M_{B} E_{B}$ (Steps 1-2) for determining the scaling.

- Finding blocks, delimited by the $i_{\ell}$ 's, of consecutive vectors in $M_{B} E_{B}$, such that typical LLL-reduction algorithms do not swap vectors between these blocks, because the $r_{i i}$ 's increase (Steps 3-4). Appropriate gaps between blocks allow to preserve the block structure after the scaling, which is a key ingredient for ensuring that $U$ is block upper-triangular, and $D^{-1} U D$ is unimodular.

- Scaling the columns of $M_{B} E_{B}$, to shrink the eventual magnitude gaps between the $r_{i i}$ 's of different blocks (Steps 5-7).

- LLL-reducing the scaled matrix (Steps 8-10).

\subsection{Correctness}

The following lemma ensures that the $\hat{r}_{i i}$ 's are good approximations of the $r_{i i}$ 's.

Lemma 14. The matrix $\widehat{R}$ computed at Step 2 of Algorithm 2 satisfies $\max _{i}\left|\hat{r}_{i i}-r_{i i}\right| / r_{i i} \leq 1 / 2$.

Proof. Thanks to the choice of $p$, Lemmata 1 and 2 ensure that $\left\|\Delta R \cdot R^{-1}\right\|_{F} \leq 2^{9} m^{3.5} \chi 2^{-p} \leq 1 / 2$, where $\Delta R=$ $\widehat{R}-R$. Looking at the diagonal coefficients of $\Delta R \cdot R^{-1}$ leads to the result.

The next part of the algorithm aims at determining the column scalings to be applied to $M_{B} E_{B}$. The scalings are computed by grouping the columns of $M_{B} E_{B}$ according to the magnitudes of the $\hat{r}_{i i}$ 's. Columns with indices in $I_{\ell}=$ $\left[i_{\ell}, i_{\ell+1}\right)$ belong to the same block. By construction, the index $i_{\ell+1}$ is the smallest $i>i_{\ell}$ such that $\min _{j>i} \hat{r}_{j j}>(8 / \theta)$. $\max _{j<i} \hat{r}_{j j}$. Let the amplitude gap between two consecutive blocks $I_{\ell-1}$ and $I_{\ell}$ be $g_{\ell}=\left(\min _{i \in I_{\ell}} \hat{r}_{i i}\right) /\left(\max _{i \in I_{\ell-1}} \hat{r}_{i i}\right)$. By construction of the blocks, and $\theta \leq 1$ (see Definition 1 ), we have $g_{\ell} \geq(8 / \theta) \geq 8$ for all $\ell$. 
At Step 7, the column scaling is set to $D=\operatorname{diag}_{i}\left(2^{d_{i}}\right)$, for each $\ell$ and each $i \in I_{\ell}$. By choice of the $d_{i}$ 's, the block structure of $M_{B} E_{B}$ is preserved for $M_{B} E_{B} D^{-1}$, but the gap between two blocks gets shrunk to at most a constant. The following result is a direct consequence of Lemma 14 and of the choice of the $d_{i}$ 's.

Lemma 15. Let $R^{\prime}$ denote the $R$-factor of $M_{B} E_{B} D^{-1}$. Then, for all $\ell$, we have $4 / 3 \leq \frac{\min _{i \geq i_{\ell}} r_{i i}^{\prime}}{\max _{i<i_{\ell}} r_{i i}^{\prime}} \leq 32$.

At Step 9 of Algorithm 2, an LLL-reduction algorithm is called. It is required that this algorithm does not interfere with the block structure. In most LLL-reduction algorithms, the only operations performed on the current lattice basis $A$ are of two types: size-reductions of vectors (an integer linear combination of basis vectors $\mathbf{a}_{j}$ with $j<i$ is subtracted from the basis vector $\mathbf{a}_{i}$ ), and swaps (two consecutive basis vectors $\mathbf{a}_{i-1}$ and $\mathbf{a}_{i}$ are exchanged). We require that swaps occur only when $r_{i, i}<r_{i-1, i-1}$. This is the case for most known LLL-reduction algorithms, including [10, 16, $14,12,15]$. We say that these LLL-reduction algorithms are well-behaved. Further, if the used LLL-reduction algorithm handles only integer matrices, we may multiply matrix $M_{B} E_{B} D^{-1}$ by a power of 2 to make it integral, and reduce the scaled matrix: the computed transformation $U$ will also be a valid LLL-reducing matrix for $M_{B} E_{B} D^{-1}$ as LLL-reducedness is invariant under basis scaling.

THEOREM 2. Assuming the LLL-reducing algorithm used at Step 9 is well-behaved (as defined just above), Algorithm 2 is correct. In particular, the matrix $D^{-1} U D$ is unimodular and the matrix $\left(M_{B} E_{B}\right)\left(D^{-1} U D\right)$ is $\Xi$-LLL-reduced.

Proof. Using Lemma 15 and the assumption on the LLLreducing algorithm used at Step 9, we obtain that the computed matrix $U$ is block-upper triangular, in the following sense. For any $\ell, \ell^{\prime}$, we define $U_{\ell \ell^{\prime}}=\left(u_{i j}\right)_{i \in I_{\ell}, j \in I_{\ell^{\prime}}}$. Then for any $\ell>\ell^{\prime}$, we have $U_{\ell \ell^{\prime}}=0$. Now, the diagonal coefficients of $D$ are non-decreasing powers of 2 , and $d_{i}=d_{j}$ when $i, j$ belong to the same $I_{\ell}$. As a result, the matrix $D^{-1} U D$ is integral: for $\ell \leq \ell^{\prime}$, submatrix $U_{\ell \ell^{\prime}}$ becomes $2^{d_{\ell^{\prime}}-d_{\ell}} \cdot U_{\ell \ell^{\prime}}$. Further, since $1=|\operatorname{det} U|=\prod_{\ell}\left|\operatorname{det} U_{\ell \ell}\right|$, we obtain that all $U_{\ell \ell}$ 's are unimodular. This implies that $D^{-1} U D$ is unimodular. It remains to show that $\left(M_{B} E_{B}\right)\left(D^{-1} U D\right)$ is $\Xi$-LLL-reduced. Let $R^{\prime}$ and $R^{\prime \prime}$ respectively denote the R-factors of $\left(M_{B} E_{B} D^{-1}\right) U$ and $\left(M_{B} E_{B}\right)\left(D^{-1} U D\right)$. We have $r_{i j}^{\prime \prime}=r_{i j}^{\prime} 2^{d_{j}}$, for all $i, j$. By $(\delta, \eta, \theta / 2)$-reducedness of $\left(M_{B} E_{B} D^{-1}\right) U$, we have, for any $i \leq j$ :

$$
\begin{aligned}
\left|r_{i j}^{\prime \prime}\right|=\left|r_{i j}^{\prime}\right| 2^{d_{j}} & \leq\left(\eta 2^{d_{j}}\right) \cdot r_{i i}^{\prime}+\left(\frac{\theta}{2} 2^{d_{j}}\right) \cdot r_{j j}^{\prime} \\
& =\left(\eta 2^{d_{j}-d_{i}}\right) \cdot r_{i i}^{\prime \prime}+\frac{\theta}{2} \cdot r_{j j}^{\prime \prime} .
\end{aligned}
$$

If $i$ and $j$ belong to the same $I_{\ell}$, then $d_{j}=d_{i}$ and the sizereduction condition of Definition 1 is satisfied. Otherwise, we have $i \in I_{\ell_{i}}$ and $j \in I_{\ell_{j}}$ for some $\ell_{i}<\ell_{j}$. Thanks to the assumption on the LLL-reducing algorithm (and noting that the R-factor of $M_{B} E_{B} D^{-1}$ is $R D^{-1}$ ), we have:

$$
\begin{aligned}
r_{j j}^{\prime} & \geq \min _{t \in I_{\ell_{j}}} r_{t t}^{\prime} \geq \min _{t \in I_{\ell_{j}}}\left(r_{t t} 2^{-d_{j}}\right) \\
& \geq \frac{2}{\theta} \max _{t \in I_{\ell_{i}}}\left(r_{t t} 2^{-d_{i}}\right) \geq \frac{2}{\theta} \max _{t \in I_{\ell_{i}}} r_{t t}^{\prime} \geq \frac{2}{\theta} r_{i i}^{\prime} .
\end{aligned}
$$

For the second inequality, we used the fact that for a wellbehaved LLL-reduction algorithm, the minimum of the Rfactor diagonal factors in a block cannot decrease. Similarly, in the fourth inequality, we used the fact that, the maximum of the R-factor diagonal factors in a block cannot increase. For the third inequality, we used the definition of the blocks and the lower bound on the gap between two blocks, and Lemma 14. As a result, we have $r_{j j}^{\prime \prime} \geq 2^{d_{j}-d_{i}} \frac{2}{\theta} r_{i i}^{\prime \prime}$, and, by (1), we obtain that $\left|r_{i j}^{\prime \prime}\right| \leq \theta r_{j j}^{\prime \prime}$. The output basis satisfies the size-reduction condition of Definition 1.

Similarly, by reducedness of $\left(M_{B} E_{B} D^{-1}\right) U$, we have:

$$
\begin{aligned}
\forall i: \delta\left(r_{i, i}^{\prime \prime}\right)^{2} & =\delta 2^{2 d_{i}}\left(r_{i, i}^{\prime}\right)^{2} \leq 2^{2 d_{i}}\left(\left(r_{i, i+1}^{\prime}\right)^{2}+\left(r_{i+1, i+1}^{\prime}\right)^{2}\right) \\
& \leq 2^{2\left(d_{i}-d_{i+1}\right)}\left(\left(r_{i, i+1}^{\prime \prime}\right)^{2}+\left(r_{i+1, i+1}^{\prime \prime}\right)^{2}\right) \\
& \leq\left(r_{i, i+1}^{\prime \prime}\right)^{2}+\left(r_{i+1, i+1}^{\prime \prime}\right)^{2}
\end{aligned}
$$

where we used the fact that $d_{i+1} \geq d_{i}$. The output basis satisfies the Lovász' conditions of Definition 1. This completes the proof of the theorem.

\subsection{Complexity analysis}

So far, we have shown that Algorithm 2 is correct. We now turn to estimating its run-time. Unless the exponents in $E_{B}$ are uncommonly huge, the dominating component of the cost is the LLL-reduction of Step 9. Our aim here is to bound the bit-size of the coefficients of the matrix $M_{B} E_{B} D^{-1}$, when this matrix is viewed as an integer matrix. The algorithm takes any floating-point lattice basis as input, but the run-time bound will depend on how close is $M_{B} E_{B}$ is to be LLL-reduced. More precisely, we consider a nonsingular matrix $\Sigma$ and a set $\Xi^{\prime}$ of valid LLL-parameters such that $\Sigma^{-1} M_{B} E_{B}$ is $\Xi^{\prime}$-LLL-reduced. Such a $\Sigma$ always exists (take $\Sigma$ such that $\Sigma^{-1} M_{B} E_{B}$ is orthonormal), but the bit-size bound to be proven will depend on the singular values of $\Sigma$. More precisely, for all $\ell$, we define $E_{\ell}$ as the $\left|I_{\ell}\right|$-dimensional subvector space of $\mathbb{R}^{m}$ that is spanned by the columns of $M_{B} E_{B}$ with indices in $I_{\ell}$. We define $F_{\ell}$ as the projection of $E_{\ell}$ orthogonally to $F_{1}+\ldots+F_{\ell-1}$, so that the column span of $M_{B} E_{B}$ is the orthogonal sum of the $F_{\ell}$ 's. Now, by orthogonality, the distortion $\Sigma$ acts independently on any of the $F_{\ell}$ 's. We let $\Sigma_{\ell}$ denote the corresponding $\left|I_{\ell}\right|$-dimensional non-singular linear map. The bit-size bound of the integer matrix $M_{B} E_{B} D^{-1}$ to be given as input to an LLL-reduction algorithm at Step 9 will involve the quantity $\log \prod \kappa\left(\Sigma_{\ell}\right)$. By orthogonality of the $F_{\ell}$ 's, the latter is bounded from above by $\log \prod_{1 \leq i \leq\lfloor n / 2\rfloor} \frac{\sigma_{i}}{\sigma_{m-i+1}}$, where $\sigma_{1} \geq \ldots \geq \sigma_{m}$ are the singular values of $\Sigma$. The following lemma provides a bound on the amplitude of the $\hat{r}_{i i}$ 's within a block.

Lemma 16. With $\alpha^{\prime}$ as in Lemma 4 (for $\Xi^{\prime}$ ), for any $\ell$, we have $\frac{\max _{i \in I_{\ell}} \hat{r}_{i i}}{\min _{i \in I_{\ell}} \hat{r}_{i i}} \leq 3\left(8 \alpha^{\prime} / \theta\right)^{\left|I_{\ell}\right|} \kappa\left(\Sigma_{\ell}\right)$.

Proof. We prove that for any $i, j \in I_{\ell}$, we have $\hat{r}_{j j} / \hat{r}_{i i} \leq$ $3\left(8 \alpha^{\prime} / \theta\right)^{\left|I_{\ell}\right|} \kappa\left(\Sigma_{\ell}\right)$. Suppose first that $j \leq i$. Then, by Lemma 4 , we have $r_{j j}^{\prime} / r_{i i}^{\prime} \leq\left(\alpha^{\prime}\right)^{i-j}$, where $R^{\prime}$ denote the R-factor of $C$. By Lemma 3, we obtain that $r_{j j} / r_{i i} \leq$ $\left(\alpha^{\prime}\right)^{i-j} \kappa\left(\Sigma_{\ell}\right)$. Finally, by Lemma 14 , we obtain that $\hat{r}_{j j} / \hat{r}_{i i} \leq$ $3\left(\alpha^{\prime}\right)^{i-j} \kappa\left(\Sigma_{\ell}\right)$. Suppose now that $j>i$. If $\hat{r}_{i i}=\max _{t \geq i} \hat{r}_{t t}$, then the bound holds since the right hand side is $\geq 1$. Otherwise, from the definition of blocks, there exists some $i^{\prime} \in I_{\ell}$ with $i^{\prime}>i$, such that $\hat{r}_{i^{\prime} i^{\prime}} \leq(8 / \theta) \cdot \hat{r}_{i i}$. Applying the same idea to $i^{\prime}$ we get $\hat{r}_{i^{\prime \prime} i^{\prime \prime}} \leq(8 / \theta)^{t} \cdot \hat{r}_{i i}$, with $i^{\prime \prime}=i_{\ell+1}-1$, where $t \leq\left|I_{\ell}\right|$ is the number of times this recursion is applied. Since $j \leq i^{\prime \prime}$, we conclude that $\hat{r}_{j j} \leq 3\left(\alpha^{\prime}\right)^{i^{\prime \prime}-j} \kappa\left(\Sigma_{\ell}\right) \hat{r}_{i^{\prime \prime} i^{\prime \prime}} \leq$ $3\left(8 \alpha^{\prime} / \theta\right)^{\left|I_{\ell}\right|} \kappa\left(\Sigma_{\ell}\right) \hat{r}_{i i}$ (using the first part of the proof).

From here, we can derive a bound on the amplitude of the diagonal coefficients of the R-factor of $M_{B} E_{B} D^{-1}$. 
Lemma 17. Let $R^{\prime}$ denote the $R$-factor of $M_{B} E_{B} D^{-1}$. Then $\frac{\max _{i} r_{i i}^{\prime}}{\min _{i} r_{i i}^{\prime}} \leq c^{n} \cdot \prod \kappa\left(\Sigma_{\ell}\right)$, for some $c$ depending only on $\Xi^{\prime}$.

Proof. Thanks to Lemma 15, we have that for all $\ell$, $\min _{i \in I_{\ell}} r_{i i}^{\prime} \leq 32 \cdot \max _{i \in I_{\ell-1}} r_{i i}^{\prime}$.

Using Lemma 14 we then translate the bound of Lemma 16 for $I_{\ell-1}$ in terms of the $r_{i i}^{\prime}$ 's rather than the $\hat{r}_{i i}$ 's, hence get: $\min _{i \in I_{\ell}} r_{i i}^{\prime} \leq 100\left(8 \alpha^{\prime} / \theta\right)^{\left|I_{\ell}\right|} \kappa\left(\Sigma_{\ell}\right) \cdot \min _{i \in I_{\ell-1}} r_{i i}^{\prime}$. Taking the product over all values of $\ell$ leads to the result.

We can now prove the following bit-size bound for the input to the LLL-reduction algorithm.

TheOrem 3. Assume that the input matrix $M_{B}$ is integral with $\left\|M_{B}\right\| \leq 2^{p}$. Let $\Sigma \in \mathbb{R}^{m \times m}$ be non-singular, such that $\Sigma^{-1} M_{B} E_{B}$ is $\Xi^{\prime}$-LLL-reduced, for some valid set $\Xi^{\prime}$ of LLL-parameters. Let $C=M_{B} E_{B} D^{-1}$ be the matrix given as input to an LLL-reduction algorithm at Step 9 of Algorithm 2. Then there exists a constant $c$ such that $C \in$ $2^{-k} \mathbb{Z}^{m \times n}$ for some $k$ satisfying:

$$
\log \|C\|-k \leq c \cdot n+2 p+2 \log \prod_{1 \leq i \leq\lfloor n / 2\rfloor} \frac{\sigma_{i}}{\sigma_{m-i+1}},
$$

where $\sigma_{1} \geq \ldots \geq \sigma_{m}$ denote the singular values of $\Sigma$.

Proof. Lemma 11 gives: $\max \left\|\mathbf{c}_{i}\right\| \leq \operatorname{cond}(C) \max r_{i i}^{\prime}$, where $R^{\prime}$ denotes the R-factor of $C$. Further, by Lemmata 7 and 9 , there exists a constant $c_{1}$ such that cond $(C) \leq \kappa(\Sigma)$. $c_{1}^{n}$. Using Lemma 17 and the fact that $\min \left\|\mathbf{c}_{i}\right\| \geq \min r_{i i}^{\prime}$, we obtain that

$$
\frac{\max \left\|\mathbf{c}_{i}\right\|}{\min \left\|\mathbf{c}_{i}\right\|} \leq c_{2}^{n} \kappa(\Sigma) \prod_{\ell} \kappa\left(\Sigma_{\ell}\right) \leq c_{2}^{n} \prod_{1 \leq i \leq\lfloor n / 2\rfloor} \frac{\sigma_{i}^{2}}{\sigma_{m-i+1}^{2}},
$$

for some constant $c_{2}$. Now, using the assumption that $M_{B}$ is integral of norm $\leq 2^{p}$, we have

$$
\frac{\max 2^{e_{i}-d_{i}}}{\min 2^{e_{i}-d_{i}}} \leq 2^{p} \cdot \frac{\max \left\|\mathbf{c}_{i}\right\|}{\min _{i}\left\|\mathbf{c}_{i}\right\|} \leq 2^{p} c_{2}^{n} \prod_{1 \leq i \leq\lfloor n / 2\rfloor} \frac{\sigma_{i}^{2}}{\sigma_{m-i+1}^{2}} .
$$

To complete the proof, we note that the entries of $C$ are sums of powers of 2 , and we use that $2^{p} \frac{\max 2^{e_{i}-d_{i}}}{\min 2^{e_{i}-d_{i}}}$ bounds from above the ratio between the smallest and the largest entries.

As our bound applies to any $\Sigma$ such that $\Sigma^{-1} M_{B} E_{B}$ is LLL-reduced, we are interested in the existence of such a $\Sigma$ with a small $\log \prod_{i \leq\lfloor n / 2\rfloor} \frac{\sigma_{i}}{\sigma_{m-i}}$.

\section{PRACTICAL CONSIDERATIONS}

Several important points deserve further investigations, especially from a practical point of view. The sharpness of the sufficient bound $2 \log \operatorname{cond}(R)+n(1+\epsilon)+O(\log m)$ on the input precision is unclear. Studying heuristic values for the different quantities to choose, e.g. for the constant $c$ of Lemma 13, remains to be done. In the same vein, understanding the impact of the chosen precision and the input basis structure on the output parameters $\Xi=(\delta, \eta, \theta)$ is an interesting problem. For an idea of practical accelerations that can be obtained thanks to the scaling, we may refer to $[1$, Se. 5$]$. A extensive experimental study should be made.
Acknowledgements. We thank Mark Watkins for pointing out the reference [5, Se. 2.6.1], and Cong Ling for valuable discussions. Part of this work was undergone while the second and third authors were visiting the University of Sydney, whose hospitality is gratefully acknowledged. This work has been supported in part by ERC Starting Grant ERC2013-StG-335086-LATTAC, and by the French National Research Agency Grant ANR-11-BS02-013-HPAC.

\section{REFERENCES}

[1] J. Bi, J.-S. Coron, J.-C. Faugère, P. Nguyen, G. Renault, and R. Zeitoun. Rounding and chaining LLL: Finding faster small roots of univariate polynomial congruences. In Proc. PKC'14, Buenos Aires, Argentina, LNCS 8383, 160-168. Springer, 2014.

[2] J. Buchmann. Reducing Lattice Bases by Means of Approximations. In Proc. ANTS, LNCS 877, 160-168. Springer, 1994.

[3] X.-W. Chang and C.C. Paige. Componentwise perturbation analyses for the QR factorization. Numer. Math., 88:319-345, 2001.

[4] X.-W. Chang, D. Stehlé, and G. Villard. Perturbation analysis of the QR factor R in the context of LLL lattice basis reduction. Math. Comp., 81(279):1487-1511, 2012.

[5] H. Cohen. A Course in Computational Algebraic Number Theory, volume 138 of Graduate Texts in Mathematics. Springer, Berlin, 1996.

[6] N. Higham. Accuracy and Stability of Numerical Algorithms. SIAM, 2nd edition, 2002.

[7] M. van Hoeij and A. Novocin. Gradual sub-lattice reduction and a new complexity for factoring polynomials. Algorithmica, 63(3):616-633, 2012. Preliminary version: Proc. LATIN, 539-553, 2010.

[8] E. Kaltofen. On the complexity of finding short vectors in integer lattices computer algebra. In Proc. EUROCAL, LNCS 162, 236-244. Springer, 1983.

[9] B. A. Lamacchia. Basis reduction algorithms and subset sum problems. Technical report, SM thesis, Massachusetts Inst. Technol, 1991.

[10] A. Lenstra, H. Lenstra, and L. Lovász. Factoring polynomials with rational coefficients. Math. Ann., 261(4):515-534, 1982.

[11] I. Morel, D. Stehlé, and G. Villard. From an LLL-reduced basis to another. ACM Commun. Comput. Algebra, 42(3):142-143, February 2009. ISSAC'08 poster.

[12] I. Morel, D. Stehlé, and G. Villard. H-LLL: Using Householder inside LLL. In Proc. ISSAC, Seoul, Republic of Korea, 271-278. ACM, 2009.

[13] H. Najafi, M. Jafari, and M.-O. Damen. On adaptive lattice reduction of correlated fading channels. IEEE Trans. Commun., 59(5):1224-1227, 2011.

[14] P. Nguyen and D. Stehlé. An LLL algorithm with quadratic complexity. SIAM J. Comput., 39(3):874-903, 2009. Preliminary version: Proc. EUROCRYPT, LNCS 3494, 215-233, 2005.

[15] A. Novocin, D. Stehlé, and G. Villard. An LLL-reduction algorithm with quasi-linear time complexity: extended abstract. In Proc. STOC, San Jose, USA, 403-412. ACM, 2011.

[16] A. Schönhage. Factorization of univariate integer polynomials by Diophantine approximation and an improved basis reduction algorithm. In Proceedings of ICALP, LNCS 172, 436-447. Springer, 1984.

[17] A. van der Sluis. Condition numbers and equilibration of matrices. Numer. Math., 14:14-23, 1969.

[18] A. Storjohann. The shifted number system for fast linear algebra on integer matrices. J. Compl., 21(4):609-650, 2005

[19] H. Zha. A componentwise perturbation analysis of the QR decomposition. SIAM J. Matrix Anal. Appl., 14(4):1124-1131, 1993. 